\title{
Encoding basic math rules
}

To solve a mathematical problem, such as which of two products is less expensive, we use abstract rules that dictate how to structure, process and evaluate numerical information. New research shows that rhesus monkeys have specific neurons that encode the abstract 'greater than' rule and others that encode the 'less than' rule. This suggests that individual cells in human brains might also encode mathematical rules.

Andreas Nieder and Sylvia Bongard of the University of Tübingen in Germany trained two rhesus monkeys to do a simple rule-based numerical task (Proc. Natl. Acad. Sci. USA published online 19 January 2010; doi:10.1073/pnas.0909180107). This task required the monkeys to compare set sizes (e.g., the number of dots in two different displays) and flexibly switch back and forth between the abstract 'greater than' and 'less than' rules.

For each of the 160 trials, the monkeys were first presented with a sample stimulus that displayed a specific number of dots. After a delay period (delay 1), the monkeys

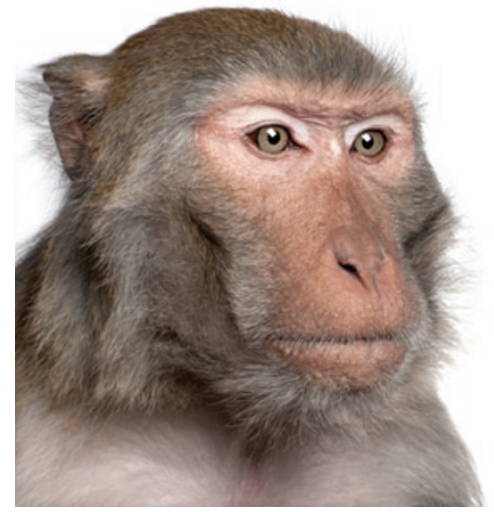

sample display. The monkeys learned the task and responded correctly most of the time. They also immediately generalized the 'greater than' and 'less than' rules for numbers that had not been previously tested, showing that they understood the basic mathematical principle.

During these tests, Nieder and Bongard recorded the activity of 484 randomly selected single neurons in the prefrontal cortex region of each monkey's brain. They found that during the second half of the

delay 2 period (after the monkeys knew the rule, but before they could know how to respond to the test display), $19 \%$ of neurons showed activity that varied significantly and exclusively with the cued rule.

These results show that, in rhesus monkeys, single prefrontal cortex neurons represent specific 'greater than' and 'less than' rules and help guide mathematical decisions. The authors explain that these results help "pave the way for a better understanding of the processing of basic mathematical rules in the primate brain."

Kirsten Dorans

\section{ACTTVATING APOPTOSTS TO TREAT RHEUMATOTD ARTHRITIS}

Rheumatoid arthritis is a chronic, destructive inflammation, primarily of the joints, that affects up to $1 \%$ of the world's population. The cause of the disease is not known, but its progression is autoimmune: immune cells called macrophages, which normally die after attacking an invader, instead persist and collect in the cartilage and bone, where their proliferation results in inflammation and joint destruction. The proliferation is thought to stem from an imbalance or deficiency in the signaling pathways for apoptosis (programmed cell death). Currently available treatments for rheumatoid arthritis include chemotherapy and steroid administration, but these are not effective in all patients and may have unwanted side effects.

Now, Harris Perlman (Northwestern University, Feinberg School of Medicine, Chicago, IL) and colleagues report that expression of Bim, a pro-apoptotic protein, is deficient in joints affected by rheumatoid arthritis and that treatment with a molecule similar to Bim protected mice against rheumatoid arthritis and even reversed the disease course in mice that had already developed rheumatoid arthritis.

The researchers first examined expression of Bim in the synovial lining of joints of people with rheumatoid arthritis. Bim expression was decreased in macrophages in these tissues, and the decrease in Bim expression was associated with an increase in inflammatory score. They found that Bim controlled the activation of macrophages and that decreased Bim expression contributed to increased macrophage activation.

Because the proliferation of macrophages contributes to the development of rheumatoid arthritis and Bim expression seems to limit macrophage proliferation, Perlman's group decided to test whether a peptide that mimics the expression of Bim (a mimetic) could interfere with the development of rheumatoid arthritis. They induced an early stage of inflammatory arthritis in mice and then treated some of them with the mimetic. Treatment with the mimetic reduced ankle circumference (an indicator of inflammation associated with arthritis), arthritis development, bone erosion and inflammation in the mice (Arthritis Rheum. 62, 441-451; 2010). They also tested the mimetic in mice with established inflammatory arthritis and found that it reduced ankle circumference and bone erosion in these mice as well. Notably, the mimetic was nontoxic and did not affect kidney or liver function in the mice.

The study results suggest that the Bim mimetic may have therapeutic potential for rheumatoid arthritis in humans. Further studies are needed to evaluate the treatment in other models of inflammatory arthritis and to develop a more precise technique for administering the mimetic. 